\title{
NUMERICAL ANALYSIS OF NONLINEAR ELLIPTIC-PARABOLIC EQUATIONS
}

\author{
EMmanuel Maitre ${ }^{1}$
}

\begin{abstract}
This paper deals with the numerical approximation of mild solutions of elliptic-parabolic equations, relying on the existence results of Bénilan and Wittbold (1996). We introduce a new and simple algorithm based on Halpern's iteration for nonexpansive operators (Bauschke, 1996; Halpern, 1967; Lions, 1977), which is shown to be convergent in the degenerate case, and compare it with existing schemes (Jäger and Kačur, 1995; Kačur, 1999).
\end{abstract}

Mathematics Subject Classification. 65M12, 35K65, 35K55, 65N22.

Received: June 15, 2001. Revised: November 16, 2001.

\section{INTRODUCTION}

Let $\Omega$ be a bounded open subset of $\mathbb{R}^{d}, d \geq 1$, and $Q=(0, T) \times \Omega$, for $T>0$. Let $b$ a continuous nondecreasing function on $\mathbb{R}$ with $b(0)=0$, and $v_{0} \in L^{1}(\Omega)$ such that $v_{0}(x) \in \overline{b(\mathbb{R})}$ a.e. on $\Omega$. For $f \in L^{1}(Q)$ we consider, from a numerical point of view, the following problem: find a function $u$ verifying

$$
(E P) \begin{cases}\frac{\partial b(u)}{\partial t}-\operatorname{div} a(u, \nabla u)=f & \text { on } Q, \\ u=0 & \text { on }(0, T) \times \partial \Omega, \\ b(u)(0)=v_{0} & \text { on } \Omega,\end{cases}
$$

where $a:(z, \xi) \in \mathbb{R} \times \mathbb{R}^{d} \rightarrow \mathbb{R}^{d}$ is continuous in $z, \xi$ and monotone in $\xi$. The fact that $b$ could become constant, brings the degeneracy from parabolic to elliptic.

This kind of equations arises naturally in a number of physical models among which are the porous medium equation, ferromagnetism hysteresis, etc. Our motivation was the study of the pressure equation in an injection moulding process, which can be written as a doubly nonlinear equation [17].

The existence of solution to $(E P)$ in this form was first established by Alt and Luckhaus [1] and Simondon [20], whereas uniqueness results were obtained more recently by Otto [19]. In [5], existence of mild solutions was obtained under weaker structure assumptions on $a$ :

$$
\begin{cases}(a(z, \xi)-a(z, 0)) \cdot \xi \geq \lambda(b(z))|\xi|^{p} & \text { for all }(z, \xi) \in \mathbb{R} \times \mathbb{R}^{d} \\ |a(z, \xi)| \leq \Lambda(z)\left(1+|\xi|^{p-1}\right) & \text { with }|\xi| \geq R(b(z)), \\ & \text { for all }(z, \xi) \in \mathbb{R} \times \mathbb{R}^{d}\end{cases}
$$

Keywords and phrases. Elliptic-parabolic, numerical, iterative method.

${ }^{1}$ Laboratoire de Mathématiques et Application, Université de Haute-Alsace, 4 rue des frères Lumière, 68093 Mulhouse Cedex, France. e-mail: E.Maitre@univ-mulhouse.fr 
where $\lambda>0, \Lambda, R \geq 0$ are continuous functions and $1<p<\infty$.

References to articles dealing with variational solutions, sometimes with different assumptions on $a$ (in particular for time dependence), can be found in the bibliography of [18].

The numerical analysis of related problems was first considered in $[4,16]$ in the case where $b^{-1}$ is Lipschitz continuous and the equation written as a porous-medium equation. This scheme was adapted to more general porous medium equations in [10,13]. More recently Jäger and Kačur [11] and Kačur [12] studied the numerical approximation of $(E P)$. However their numerical scheme was shown to converge for strictly increasing and Lipschitz continuous $b$, or in the particular case where $a$ depends on $u$ through $b(u)$.

In this article we introduce a numerical scheme for the case where $b$ could become constant, but remains between two strictly increasing linear functions outside some compact set (see Rem. 1.1):

$(H 2) b$ is a locally Lipschitz function on $\mathbb{R}$ and

$$
\exists K \geq 0, \exists(\ell, L), 0<\ell \leq L, \forall r \in \mathbb{R},|r| \geq K, \min (\ell r, L r) \leq b(r) \leq \max (\ell r, L r) .
$$

Note that under this assumption $b$ can still become constant on some open set. However the constant steps have to be of bounded length.

Remark 1.1. For example if we consider $b(r)=0$ on $[-1,1], b(r)=r-1$ on $[1,+\infty[$ and $b(r)=r+1$ on ] $-\infty,-1]$ (H2) is verified with $K=2, L=1, \ell=\frac{1}{2}$. However both $b(r)=\operatorname{sgn}(r) \sqrt{|r|}$ and $b(r)=\max (r, 0)$, fail to verify $(H 2)$, the first one because it is non-Lipschitz in zero, the second one because it does not remain between to strictly increasing linear functions in the neighborhood of $-\infty$.

\section{Existence OF Mild SOLUTiOnS}

Note that under assumptions $(H 1)$ and $(H 2)$, the existence of weak solutions is an open question, although some progress in that direction have been made recently [6] in dimension one. The good notion of solution there, is the notion of mild solutions since it is proved in [5] that there exists an exact mild solution $u$ of $(E P)$.

For an integer $N$ we denote by $t_{n}=n h, n=0, \ldots, N$ the subdivision of $[0, T]$ in $N$ small intervals of length $h=\frac{T}{N}$ (see the remark below explaining why we work with a constant step subdivision). An exact mild solution of $(E P)$ is a measurable function $u$ verifying $v=b(u) \in C\left([0, T] ; L^{1}(\Omega)\right), v(0)=v_{0}$ and, for any $\varepsilon>0$, there exists $N \in \mathbb{N}$ such that for any

$$
\begin{gathered}
f_{1}, \ldots, f_{N} \in L^{\infty}(\Omega) \text { such that } \sum_{n} \int_{t_{n}}^{t_{n+1}}\left\|f(t)-f_{n+1}\right\|_{1} \mathrm{~d} t \leq h, \\
u_{0} \in L^{\infty}(\Omega) \text { such that }\left\|v_{0}-b\left(u_{0}\right)\right\|_{1} \leq h,
\end{gathered}
$$

there exists $\left(u_{1}, \ldots, u_{N}\right)$ verifying for $n=0, \ldots, N-1$

$$
\left\{\begin{array}{l}
\frac{b\left(u_{n+1}\right)-b\left(u_{n}\right)}{h}-\operatorname{div} a\left(u_{n+1}, \nabla u_{n+1}\right)=f_{n+1} \text { in } \mathcal{D}^{\prime}(\Omega) \\
u_{n+1} \in W_{0}^{1, p}(\Omega) \cap L^{\infty}(\Omega)
\end{array}\right.
$$

and such that $\left\|v(t)-b\left(u_{n+1}\right)\right\|_{1} \leq \varepsilon$ for any $t \in\left(t_{n}, t_{n+1}\right], n=0, \ldots, N-1$.

Remark 2.1. The exact mild solution verifies more than the preceding, since the time subdivision needs not to be of constant step. It could be useful for an adaptative time step numerical scheme (see the final discussion and conclusion). However the technics developed thereafter can be adapted to a varying step subdivision without difficulty. So we chose for sake of readability to present the constant step algorithm. 


\section{Existing SCHEMES FOR RELATED PROBLEMS}

The idea in [11] (under their assumptions a weak solution does exist) is basically to approach the solution of the equivalent equation

$$
b^{\prime}(u) u_{t}-\operatorname{div} a(u, \nabla u)=f
$$

by the following scheme: for $N$ integer and $h=\frac{T}{N}$ we denote by $u_{n}$ the approximation of $u\left(t_{n}\right)$. Given $u_{n}$, to get $u_{n+1}$ one first compute the solution $\theta_{n+1}$ of the elliptic problem

$$
\lambda_{n}\left(\theta_{n+1}-u_{n}\right)-h \operatorname{div} a\left(u_{n}, \nabla \theta_{n+1}\right)=h f\left(t_{n+1}\right)
$$

with the Dirichlet boundary condition, where $\lambda_{n} \in L^{\infty}(\Omega)$ should verify

$$
\left\|\lambda_{n}-\frac{b_{d}\left((1-\alpha) u_{n}+\alpha \theta_{n+1}\right)-b_{d}\left(u_{n}\right)}{\theta_{n+1}-u_{n}}\right\|_{\infty}<h .
$$

Then $u_{n+1}$ is obtained by

$$
u_{n+1}=(1-\alpha) u_{n}+\alpha \theta_{n+1} .
$$

The function $b_{d}$ is a regularization of $b: b_{d}(s)=b(s)+h^{d} s ; d \in\left(0, \frac{1}{2}\right)$ and $\alpha$ close to 1 are the parameters of the method. The coefficient $\lambda_{n}$ is obtained eventually thanks to an iteration

$$
\lambda_{n}^{k+1}=\frac{b_{d}\left((1-\alpha) u_{n}+\alpha \theta_{n+1}^{k}\right)-b_{d}\left(u_{n}\right)}{\theta_{n+1}^{k}-u_{n}}
$$

where $\theta_{n+1}^{k}$ is obtained as the solution of (3) with $\lambda_{n}=\lambda_{n}^{k}$. This algorithm is proved to converge when $b$ is strictly increasing and Lipschitz continuous. The numerical scheme we present now is stable even when $b^{\prime}$ is identically zero on some open set, or does not satisfy a global Lipschitz condition (see $(H 2)$ ).

\section{Numerical ALGORIthm}

Given $f_{1}, \ldots, f_{N}, u_{0}$, we are now addressing the problem of the numerical resolution of (1). We point out that the initial condition in $(E P)$ is in fact given on $b(u)$ instead of $u$ for a parabolic problem. This is because in $(E P)$, in general, one has only the time continuity of $b(u)$. If $b$ has some constant steps in its graph, then the initial condition on $u$ can be undetermined.

For the same reason, the explicit scheme

$$
\left\{\begin{array}{l}
\frac{b\left(u_{n+1}\right)-b\left(u_{n}\right)}{h}-\operatorname{div} a\left(u_{n}, \nabla u_{n}\right)=f_{n+1}, \quad n=0, \ldots, N-1 \\
b\left(u_{0}\right)=v_{0},
\end{array}\right.
$$

could easily be solve in $v_{n+1}:=b\left(u_{n+1}\right)$ but nothing could prevent $v_{n+1}$ from going out of the range of $b$. Then $u_{n+1}$ could not be recovered. And even if $v_{n+1}$ remains in the range of $b$, its degeneracy could bring a bad numerical behavior.

So we have to solve the implicit scheme directly in $u$. For this we introduce the following iterative process to get $u_{n+1}$ from $u_{n}$ :

$$
\left\{\begin{array}{l}
\text { Let } u_{n+1}^{0} \in L^{\infty}(\Omega)\left(\text { e.g. } u_{n}\right), \text { solve for } k=0,1, \ldots, \\
u_{n+1}^{k+1}-\rho \operatorname{div} a\left(u_{n+1}^{k+1}, \nabla u_{n+1}^{k+1}\right)=\lambda_{k} u_{n+1}^{k}-\frac{\rho}{h}\left(b\left(\lambda_{k} u_{n+1}^{k}\right)-b\left(u_{n}\right)\right)+\rho f_{n+1}
\end{array}\right.
$$


where $\rho>0$ is a given parameter, and $\left(\lambda_{k}\right)$ is a sequence of $] 0,1[$ such that

$$
\lim _{k \rightarrow \infty} \lambda_{k}=1, \quad \prod_{k \geq 0} \lambda_{k}=0, \quad \sum_{k \geq 0}\left|\lambda_{k+1}-\lambda_{k}\right|<\infty
$$

For example $\lambda_{k}=1-\frac{1}{k+1}$ is a convenient choice. Note that the introduction of $\lambda_{k}$ in this scheme is an application of the ideas of Halpern [9] sharpened by Lions [15] and more recently by Bauschke [2]. Indeed, in the interesting case, where $b$ can degenerate, the iteration $(S)$ with $\lambda_{k}=1$ is non-expansive but not strictly contractant.

Remark 4.1. Let us compare $(S)$ (with $\lambda_{k}=1$ ) with the scheme of Jäger and Kačur that we recalled before, in the simpler case where $\alpha=1$ and without regularization of $b$ : in this case we have $\theta_{n+1}=u_{n+1}$ and $\lambda_{n}^{k+1}=\frac{b\left(u_{n+1}^{k}\right)-b\left(u_{n}\right)}{u_{n+1}^{k}-u_{n}}$ and we make the following iteration to get $u_{n+1}^{k+1}$ from $u_{n+1}^{k}$ :

$$
\lambda_{n}^{k+1}\left(u_{n+1}^{k+1}-u_{n}\right)-h \operatorname{div} a\left(u_{n}, \nabla u_{n+1}^{k+1}\right)=h f\left(t_{n+1}\right) .
$$

Setting $\rho_{n}^{k+1}=\frac{h}{\lambda_{n}^{k+1}}$ we get

$$
u_{n+1}^{k+1}-\rho_{n}^{k+1} \operatorname{div} a\left(u_{n}, \nabla u_{n+1}^{k+1}\right)=u_{n}+\rho_{n}^{k+1} f\left(t_{n+1}\right) .
$$

Thus our scheme appears as an analog of this scheme, with a correction term in the right-hand side. The first advantage, from the computational point of view, is that there is no need to compute these $\lambda_{n}^{k+1}$ for which one has to test whether $u_{n+1}^{k}=u^{n}$ or not. Another point is that this scheme could exhibit a bad numerical behavior at times where $u(t)$ is discontinuous. Indeed, solutions $u$ of $(E P)$ need not to be continuous in time. Only $b(u)$ have to. If such a discontinuity happens, the estimation of the derivative of $b$ in $\lambda_{n}^{k+1}$ is nonsense. On contrary numerical tests we made (see at the end of article) show that our method works even in case of discontinuous $u$.

\section{Existence of A SOlution to $(S)$ Under $(H 1)-(H 2)$}

First we prove that $(S)$ has a solution $u_{n+1}^{k+1} \in W_{0}^{1, p}(\Omega) \cap L^{\infty}(\Omega)$. Indeed one has $u_{n+1}^{0}=u_{n} \in W_{0}^{1, p}(\Omega) \cap$ $L^{\infty}(\Omega)$ from (1). Assuming $u_{n+1}^{k} \in W_{0}^{1, p}(\Omega) \cap L^{\infty}(\Omega)$ we have from the assumptions on $b$, and the definition of mild solution, $g:=\lambda_{k} u_{n+1}^{k}-\frac{\rho}{h}\left(b\left(\lambda_{k} u_{n+1}^{k}\right)-b\left(u_{n}\right)\right)+\rho f_{n+1} \in L^{\infty}(\Omega)$. Let $C=\|g\|_{\infty}$, there exists a solution $w \in W_{0}^{1, p}(\Omega)$ of

$$
w-\rho \operatorname{div} a\left(T_{C}(w), \nabla w\right)=g
$$

where $T_{C}(r):=\min (C, \max (r,-C))$ denotes the standard truncature operator. Indeed now thanks to assumption $(H 1), w \rightarrow w-\rho \operatorname{div} a\left(T_{C}(w), \nabla w\right)$ is pseudo-monotone, coercive and bounded on $W_{0}^{1, p}(\Omega)$, thus surjective (see [14], p. 180).

The following lemma is a particular case of Lemma 2.5 of [5].

Lemma 5.1. Assume a verifies $(H 1)$. Let $w \in W_{0}^{1, p}(\Omega), C, \sigma>0$ and suppose that $w-\sigma \operatorname{div} a\left(T_{C}(w), \nabla w\right)=g$ with $g \in L^{\infty}(\Omega)$. Then $\|w\|_{\infty} \leq\|g\|_{\infty}$.

From this lemma we see that $\|w\|_{\infty} \leq\|g\|_{\infty}=C$ thus $w=T_{C}(w)$ verifies $w-\rho \operatorname{div} a(w, \nabla w)=g$. Setting $u_{n+1}^{k+1}=w$ we found a solution to $(S)$ belonging to $W_{0}^{1, p}(\Omega) \cap L^{\infty}(\Omega)$. 


\section{Convergence}

To prove the convergence of the whole sequence $\left(u_{n+1}^{k+1}\right)_{k \geq 0}$, we assume:

$$
(U C) \quad\left\{\begin{array}{l}
\forall f \in L^{\infty}(\Omega) \text { there is uniqueness of a solution of } \\
u \in W_{0}^{1, p}(\Omega) \cap L^{\infty}(\Omega), \quad b(u)-\operatorname{div} a(u, \nabla u)=f \quad \text { in } \mathcal{D}^{\prime}(\Omega) .
\end{array}\right.
$$

Remark 6.1. For example if $u \rightarrow-\operatorname{div} a(u, \nabla u)$ is strongly monotone then this condition is verified for all $b$.

We begin by the following lemma which provides a crucial $L^{\infty}$ uniform bound for the $u_{n+1}^{k+1}$ :

Lemma 6.2. If $\rho<\frac{h}{L}$, there exists $M>0$ independent of $k$ such that $\left\|u_{n+1}^{k}\right\|_{\infty} \leq M$.

Proof. Let $M=\max \left(\left\|u_{n+1}^{0}\right\|_{\infty}, \frac{1}{\ell}\left\|h f_{n+1}+b\left(u_{n}\right)\right\|_{\infty}, \frac{2 K}{1-\frac{\rho \ell}{h}}, \frac{2 \frac{\rho}{h}}{1-\frac{\rho \ell}{h}} \max _{[-K, K]}|b|\right)$ where $K$ is defined in (H2). By induction, we first note that $\left\|u_{n+1}^{0}\right\| \leq M$, and assume that $\left\|u_{n+1}^{k}\right\|_{\infty} \leq M$. As $u_{n+1}^{k+1} \in L^{\infty}(\Omega)$, it verifies

$$
u_{n+1}^{k+1}-\rho \operatorname{div} a\left(T_{C_{k}}\left(u_{n+1}^{k+1}\right), \nabla u_{n+1}^{k+1}\right)=\lambda_{k} u_{n+1}^{k}-\frac{\rho}{h}\left(b\left(\lambda_{k} u_{n+1}^{k}\right)-b\left(u_{n}\right)\right)+\rho f_{n+1}
$$

with $C_{k}=\left\|u_{n+1}^{k+1}\right\|_{\infty}$. Thus from Lemma 5.1,

$$
\begin{aligned}
\left\|u_{n+1}^{k+1}\right\|_{\infty} & \leq\left\|\lambda_{k} u_{n+1}^{k}-\frac{\rho}{h}\left(b\left(\lambda_{k} u_{n+1}^{k}\right)-b\left(u_{n}\right)\right)+\rho f_{n+1}\right\|_{\infty} . \\
& \leq\left\|\lambda_{k} u_{n+1}^{k}-\frac{\rho}{h} b\left(\lambda_{k} u_{n+1}^{k}\right)\right\|_{\infty}+\frac{\rho}{h}\left\|h f_{n+1}+b\left(u_{n}\right)\right\|_{\infty} .
\end{aligned}
$$

If $\left|\lambda_{k} u_{n+1}^{k}(x)\right| \leq K$ then $\left|\lambda_{k} u_{n+1}^{k}(x)-\frac{\rho}{h} b\left(\lambda_{k} u_{n+1}^{k}\right)(x)\right| \leq K+\frac{\rho}{h} \max _{[-K, K]}|b| \leq\left(1-\frac{\rho \ell}{h}\right) \frac{M}{2}+\left(1-\frac{\rho \ell}{h}\right) \frac{M}{2}=$ $\left(1-\frac{\rho \ell}{h}\right) M$ from the definition of $M$. Else as $b$ verifies $(H 2)$, and $1-\frac{\rho L}{h} \geq 0$, we get

$$
\left|\lambda_{k} u_{n+1}^{k}(x)-\frac{\rho}{h} b\left(\lambda_{k} u_{n+1}^{k}\right)(x)\right| \leq\left(1-\frac{\rho \ell}{h}\right)\left|\lambda_{k} u_{n+1}^{k}(x)\right| \leq\left(1-\frac{\rho \ell}{h}\right) M .
$$

Thus $\left\|u_{n+1}^{k+1}\right\|_{\infty} \leq\left(1-\frac{\rho \ell}{h}\right) M+\frac{\rho}{h}\left\|h f_{n+1}+b\left(u_{n}\right)\right\|_{\infty} \leq\left(1-\frac{\rho \ell}{h}\right) M+\frac{\rho \ell}{h} M=M$ using the definition of $M$.

From $(H 2)$ and $M$ defined in the proof, we note $L_{M}$ the Lipschitz constant of $b$ on $[-M, M]$. Then we have the following convergence result:

Theorem 6.3. Assume that (H1)(H2) and (UC) hold. Then for $\rho<\min \left(\frac{h}{L}, \frac{2 h}{L_{M}}\right)$ the iterative scheme $(S)$ converges, i.e.

$$
\lim _{k \rightarrow \infty} u_{n+1}^{k}=u_{n+1} \quad \text { strongly in } L^{1}(\Omega)
$$

where $u_{n+1}$ verifies (1).

Proof. Thanks to the above lemma, we can write $(S)$ as

$$
\frac{1}{\lambda_{k+1}} \bar{u}_{n+1}^{k+1}-\rho \operatorname{div} a\left(T_{M}\left(\frac{1}{\lambda_{k+1}} \bar{u}_{n+1}^{k+1}\right), \nabla \frac{1}{\lambda_{k+1}} \bar{u}_{n+1}^{k+1}\right)=\bar{u}_{n+1}^{k}-\frac{\rho}{h}\left(b\left(\bar{u}_{n+1}^{k}\right)-b\left(u_{n}\right)\right)+\rho f_{n+1},
$$

where we put $\bar{u}_{n+1}^{k}=\lambda_{k} u_{n+1}^{k}$ and $\bar{u}_{n+1}^{k+1}=\lambda_{k+1} u_{n+1}^{k+1}$. Let $A(u)=-\operatorname{div} a\left(T_{M}(u), \nabla u\right)$. 
Lemma 6.4. Let $\rho \leq \frac{2 h}{L_{M}}$, and $C_{M}=\left\{u \in L^{1}(\Omega), \quad\|u\|_{\infty} \leq M\right\}$. The iteration operator

$$
T(\bar{u})=(I+\rho A)^{-1}\left(\bar{u}-\frac{\rho}{h}\left(b(\bar{u})-b\left(u_{n}\right)\right)+\rho f_{n+1}\right)
$$

is a $L^{1}$-non-expanding operator from $C_{M}$ to $C_{M}$.

Proof. The fact that $T$ maps $C_{M}$ to $C_{M}$ is easily seen in the proof of the preceding lemma where $\lambda_{k}$ has to be replaced by 1 . Now let $(\bar{u}, \bar{v}) \in C_{M}^{2}$. One has from the T-accretiveness of $A$ in $L^{1}(\Omega)$ (see [5], Prop. 2.4 with $b(r)=r)$ that $(\mathrm{id}+\rho A)^{-1}$ is a $T$-contraction in $L^{1}(\Omega)$ thus a contraction. This entails

$$
\|T(\bar{u})-T(\bar{v})\|_{1} \leq\left\|\bar{u}-\bar{v}-\frac{\rho}{h}(b(\bar{u})-b(\bar{v}))\right\|_{1} .
$$

If $\bar{u}(x)-\bar{v}(x) \geq 0$ then as $b$ is non-decreasing and Lipschitz on $[-M, M]$, one has

$$
0 \leq b(\bar{u}(x))-b(\bar{v}(x)) \leq L_{M}(\bar{u}(x)-\bar{v}(x))
$$

thus

$$
\left(1-\frac{\rho L_{M}}{h}\right)(\bar{u}(x)-\bar{v}(x)) \leq \bar{u}(x)-\bar{v}(x)-\frac{\rho}{h}(b(\bar{u}(x))-b(\bar{v}(x))) \leq \bar{u}(x)-\bar{v}(x) .
$$

For $\rho \leq \frac{2 h}{L_{M}}$, one gets $\left|\bar{u}(x)-\bar{v}(x)-\frac{\rho}{h}(b(\bar{u}(x))-b(\bar{v}(x)))\right| \leq|\bar{u}(x)-\bar{v}(x)|$. The case $\bar{u}(x)-\bar{v}(x) \leq 0$ is symmetric. Upon integration on $\Omega$ one ends with

$$
\|T(\bar{u})-T(\bar{v})\|_{1} \leq\|\bar{u}-\bar{v}\|_{1} .
$$

Thus one has the iteration $\bar{u}_{n+1}^{k+1}=\lambda_{k+1} T\left(\bar{u}_{n+1}^{k}\right)$ where $T$ is a non-expansive operator in $L^{1}(\Omega)$. The following lemma proves the convergence of this iteration under our assumptions. It is an easy adaptation and weaker form of several known results $[2,9,15]$, but which has the advantage to be applicable in a non-reflexive Banach space.

Lemma 6.5. Let $X$ be a Banach space and $C$ a convex subset of $X$, containing 0 . Let $T$ be a non-expansive operator on $C$ such that $T(C) \subseteq C$, admitting a unique fixed point $x^{*}$ in $C$. Let $\lambda_{k}$ be a sequence of $] 0,1[$ verifying (4). Then the sequence $\left(x^{k}\right)$ generated by the iterative scheme

$$
x^{0} \in C, \quad x^{k+1}=\lambda_{k+1} T\left(x^{k}\right)
$$

verifies $\lim _{k \rightarrow \infty} x^{k}-T\left(x^{k}\right)=0$. Consequently, if all subsequences of $\left(x^{k}\right)$ have in turn a subsequence converging to a point of $C$, then the whole sequence $\left(x^{k}\right)$ converges toward $x^{*}$.

Proof. First one notes that as $0 \in C, T(C) \subseteq C$, and $x^{k+1}=\left(1-\lambda_{k+1}\right) 0+\lambda_{k+1} T\left(x^{k}\right)$, one has by induction that $x^{k}$ belongs to $C$ for all $k \in \mathbb{N}$. Moreover, the sequence $\left(x^{k}\right)$ is bounded. Indeed let $y^{k+1}$ be defined by

$$
y^{0}=x^{0}, \quad y^{k+1}=\left(1-\lambda_{k+1}\right) x^{0}+\lambda_{k+1} T\left(y^{k}\right)
$$

then $y^{k+1}-x^{*}=\left(1-\lambda_{k+1}\right)\left(x^{0}-x^{*}\right)+\lambda_{k+1}\left(T\left(y^{k}\right)-T\left(x^{*}\right)\right)$, thus $\left\|y^{k+1}-x^{*}\right\| \leq\left(1-\lambda_{k+1}\right)\left\|x^{0}-x^{*}\right\|+\lambda_{k+1}\left\|y^{k}-x^{*}\right\|$ and by induction $\left\|y^{k+1}-x^{*}\right\| \leq\left\|x^{0}-x^{*}\right\|$. Next $\left\|y^{k}-x^{k}\right\| \leq\left\|x^{0}\right\|$ for all $k$, since this is true for $k=0$ and $y^{k+1}-x^{k+1}=\left(1-\lambda_{k+1}\right) x^{0}+\lambda_{k+1}\left(T\left(y^{k}\right)-T\left(x^{k}\right)\right)$ thus $\left\|y^{k+1}-x^{k+1}\right\| \leq\left(1-\lambda_{k+1}\right)\left\|x^{0}\right\|+\lambda_{k+1}\left\|y^{k}-x^{k}\right\| \leq$ $\left(1-\lambda_{k+1}\right)\left\|x^{0}\right\|+\lambda_{k+1}\left\|x^{0}\right\|=\left\|x^{0}\right\|$. Collecting these two estimations, we prove that $\left(x^{k}\right)$ is bounded.

As $T$ is non-expansive, there exists $c>0$ such that

$$
\left\|x^{k}\right\| \leq c, \quad\left\|T\left(x^{k}\right)\right\| \leq c, \quad \forall k \in \mathbb{N} .
$$


Now since $x^{k+1}-T\left(x^{k}\right)=\left(\lambda_{k+1}-1\right) T\left(x^{k}\right)$, and $\lambda_{k} \rightarrow 1$ one has

$$
\lim _{k \rightarrow \infty} x^{k+1}-T\left(x^{k}\right)=0 .
$$

Let us prove that $x^{k+1}-x^{k} \rightarrow 0$. We write

$$
\begin{aligned}
x^{k+1}-x^{k} & =\lambda_{k+1} T\left(x^{k}\right)-\lambda_{k} T\left(x^{k-1}\right) \\
& =\left(\lambda_{k+1}-\lambda_{k}\right) T\left(x^{k-1}\right)+\lambda_{k+1}\left(T\left(x^{k}\right)-T\left(x^{k-1}\right)\right) .
\end{aligned}
$$

Passing to the norms gives $\left\|x^{k+1}-x^{k}\right\| \leq c\left|\lambda_{k+1}-\lambda_{k}\right|+\lambda_{k+1}\left\|x^{k}-x^{k-1}\right\|$. By induction one gets for all $(k, m) \in \mathbb{N}^{2}$,

$$
\left\|x^{k+1}-x^{k}\right\| \leq c \sum_{i=m+1}^{k}\left|\lambda_{i+1}-\lambda_{i}\right|+\left\|x^{m+1}-x^{m}\right\| \prod_{i=m+1}^{k} \lambda_{i+1} .
$$

Thus passing to the limsup gives, thanks to the convergences in (4),

$$
\limsup _{k \rightarrow \infty}\left\|x^{k+1}-x^{k}\right\| \leq c \sum_{i=m+1}^{+\infty}\left|\lambda_{i+1}-\lambda_{i}\right|+2 c \prod_{i=m+1}^{+\infty} \lambda_{i+1},
$$

for each $m \in \mathbb{N}$. From the properties of the sequence $\left(\lambda_{k}\right)$,

$$
\lim _{m \rightarrow \infty} \sum_{i=m+1}^{+\infty}\left|\lambda_{i+1}-\lambda_{i}\right|=0, \quad \lim _{m \rightarrow \infty} \prod_{i=m+1}^{+\infty} \lambda_{i+1}=0
$$

thus one get the convergence of the whole sequence, i.e.

$$
\lim _{k \rightarrow \infty}\left\|x^{k+1}-x^{k}\right\|=0
$$

Now since $x^{k+1}-T\left(x^{k}\right) \rightarrow 0$, we have

$$
\lim _{k \rightarrow \infty} x^{k}-T\left(x^{k}\right)=0 .
$$

To show the last assertion, note that a converging subsequence of $\left(x^{k}\right)$, still verifies $x^{k^{\prime}}-T\left(x^{k^{\prime}}\right) \rightarrow 0$. As $T$ is continuous this subsequence must converge toward a fixed point of $T$. As $x^{*}$ is the only fixed point in $C$, we have that every subsequence of $\left(x^{k}\right)$ has a subsequence converging to $x^{*}$. That entails the convergence of the whole sequence $\left(x^{k}\right)$ (if not there exists an $\varepsilon_{0}>0$ and a subsequence $\left(x^{k^{\prime}}\right)$ such that $\left\|x^{k^{\prime}}-x^{*}\right\|>\varepsilon_{0}$. Extracting a subsequence of $x^{k^{\prime}}$ converging to $x^{*}$ leads to a contradiction).

We are going to apply the previous lemma with $X=L^{1}(\Omega)$ and $C=C_{M}$ which is clearly a convex subset of $L^{1}(\Omega)$ containing 0 . Our operator $T$ is defined in Lemma 6.4. The uniqueness of fixed point is condition $(U C)$. Indeed a fixed point $u^{*}$ of $T$ verifies

$$
u^{*}-\rho \operatorname{div} a\left(T_{M}\left(u^{*}\right), \nabla u^{*}\right)=u^{*}-\frac{\rho}{h}\left(b\left(u^{*}\right)-b\left(u_{n}\right)\right)+\rho f_{n+1}
$$

thus $b\left(u^{*}\right)-h \operatorname{div} a\left(T_{M}\left(u^{*}\right), \nabla u^{*}\right)=b\left(u_{n}\right)+h f_{n+1}$. From $(U C)$ this equation has at most one solution and from the definition of mild solution it is $u_{n+1}$.

To conclude the proof of convergence of $(S)$, we point out that each subsequence of $\bar{u}_{n+1}^{k}$ has a convergent subsequence to an element of $C_{M}$, using the $L^{\infty}$ bound of $\bar{u}_{n+1}^{k}$ and the coercivity assumption (H1), to the equation $\left(S^{\prime}\right)$. Applying Lemma 6.5 to our situation, we conclude that the sequence $\bar{u}_{n+1}^{k}$ converges strongly in $L^{1}(\Omega)$ toward $u_{n+1}$. The same occurs for $u_{n+1}^{k}=\frac{1}{\lambda_{k}} \bar{u}_{n+1}^{k}$. 

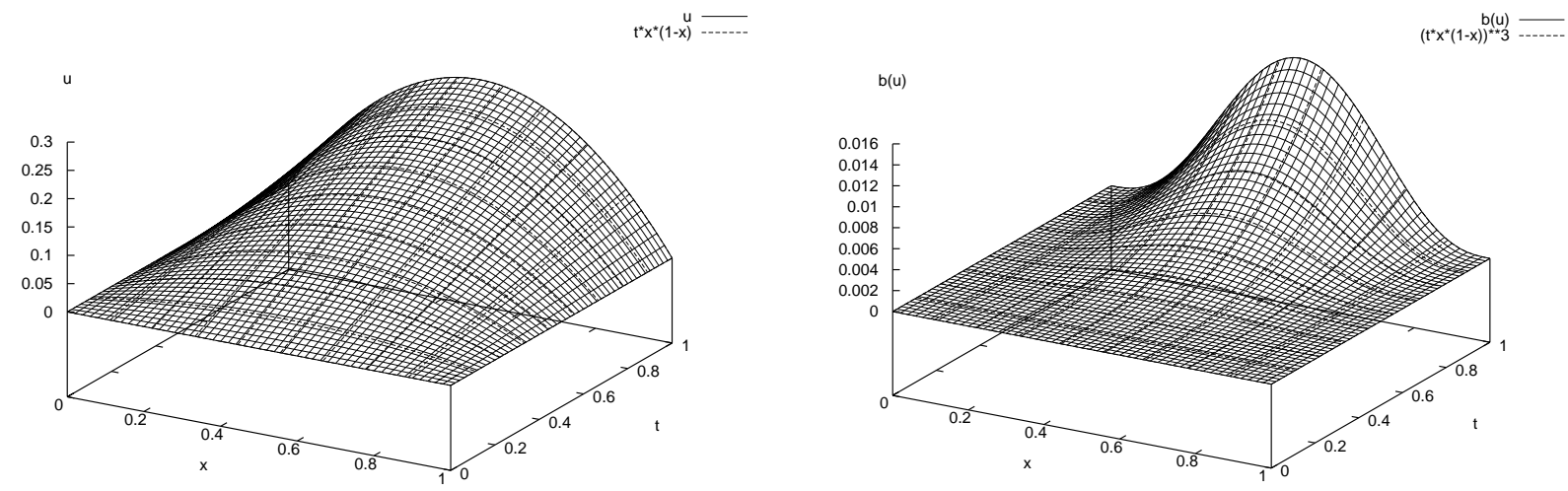

Figure 1. Test 1: $b(u)=u^{3}, f(t, x)=3 t^{2} x^{2}(1-x)^{2}+2 t, v^{0}=0$.

\section{Convergence When $h \rightarrow 0$ toward a mild solution of $(E P)$}

Note that in [11], an iteration on $k$ is performed until convergence to get the right coefficient $\lambda_{n}$ in (3). Each iteration solves a linear scheme. When $u_{n+1}$ is known, they prove the convergence of the step function $u_{N}(t)=u_{n+1}$ on $\left(t_{n}, t_{n+1}\right]$ toward the weak solution $u(t)$ as the time step goes to zero.

Under our assumptions on $a$ and $b$, the existence of a weak solution is still an open question (see [6] for one dimension). For a mild solution we do not need to show the convergence in time since it is included in its definition: once convergence in $k$ is achieved for $u_{n+1}$, then by definition of mild solution $b\left(u_{n+1}\right)$ approaches $b(u)$ on $\left(t_{n}, t_{n+1}\right.$ ] up to $\varepsilon$. If $b$ is non-degenerate, as in [11], this gives information on the behavior of $u_{n+1}$. On the contrary, note that if $b=0$ we do not get a real convergence result; the point is that in this case, from the definition of [5], every measurable function $u$ is a mild solution!

Note however that under some additional assumptions on $a$, it is proved in [5] that there exists a weak solution $w$ of $(E P)$ such that $b(w)=b(u)$ a.e. We thus have in this case the convergence of our scheme (in the same meaning) toward this weak solution.

\section{Numerical TESTS}

The stop criterium for iterations, in all the following tests is $\frac{\left\|u^{k+1}-u^{k}\right\|_{2}}{\left\|u^{k+1}\right\|_{2}} \leq 10^{-6}$ where $\|\cdot\|_{2}$ is the Euclidean norm and $u^{k}$ the vector approaching, at iteration $k$, the space-discretization of $u$.

Our first numerical test is to compare the solution given by our scheme to an exact solution. We consider in dimension 1 the case $b(u)=u^{3}, a(u, \nabla u)=u_{x}, \Omega=(0,1)$ with $f(t, x)=3 t^{2} x^{2}(1-x)^{2}+2 t$ and $v^{0}=0$, which gives as exact solution $u_{e}(t, x)=t x(1-x)$. Of course $b$ is only locally Lipschitz, but as $u$ remains bounded for bounded times, our scheme will still converge. Note that this problem is degenerate at $t=0$ since $b^{\prime}(0)=0$. For $h=0.02, \rho=0.01$ and a time step of 0.02 we plot the solutions $u, v=b(u)$ of our scheme superposed with their analytic counterparts, in Figure 1, and the corresponding errors in Figure 2.

The second numerical test is to emphasize the ability of our scheme to converge for really degenerated $b$. We chose $b(u)=0$ for $|u| \leq 1, b(u)=u-1$ for $u>1$ and $b(u)=u+1$ for $u<-1$, with $u_{0}(x)=\frac{1}{2} x(1-x)$ (thus $\left.v_{0}(x)=b\left(u_{0}(x)\right)=0\right)$ and $f(x, t)=10 t+1$.

Note that the value of $u_{0}$ plays no role, we just take this value so that it solves the equation at $t=0$, ensuring a continuity of $u$. Here we are in a totally degenerate case since we start in a zone where $b$ is identically zero. One see that $u$ increases (in time) as $f$ increases whereas $b$ remains zero until $u$ reach 1 . The time for which $\|u(t)\|_{\infty}$ reaches 1 can be computed explicitly and is $t_{c}=0.7$. Thus for $t<t_{c}$, we solve a pure elliptic problem and starting from $t=t_{c}$ we solve a parabolic problem. We can see in Figure 3 that $t_{c}$ is well computed.

In the two previous cases, $u$ was continuous in time (and space). We compared our method with Kačur algorithm, and found that the two methods give exactly the same results. For the parameters of Kačur algorithm, 

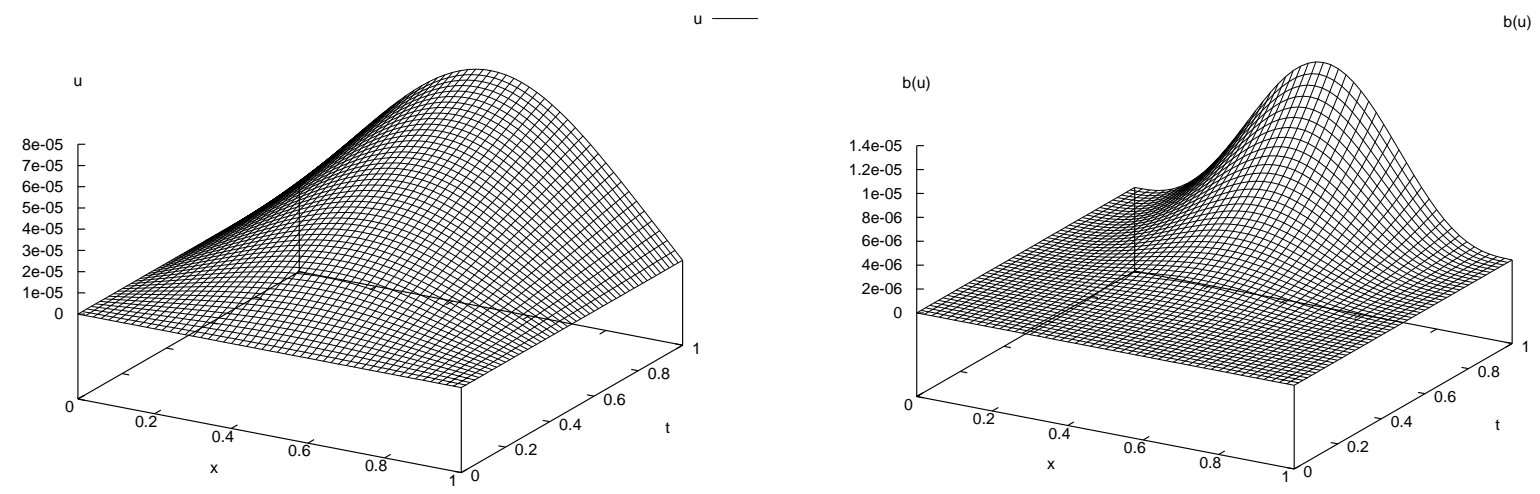

Figure 2. Test 1: Error $u-u_{e}$ and $b(u)-b\left(u_{e}\right)$.
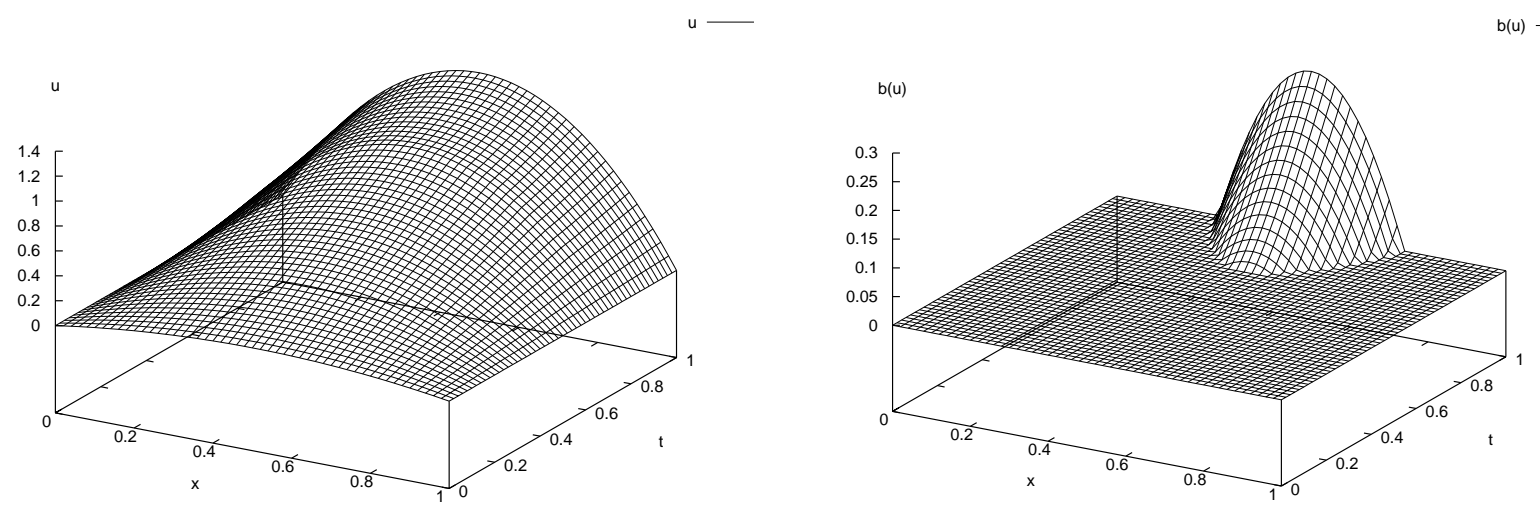

Figure 3. Test 2: Degenerate case $b(u)=0$ for $|u| \leq 1$ and linear outside.

we took $\alpha=1$, and $d=5$ (see [11]). Note that $d=5$ is not permitted theoretically, but it works here and gave better results than $d=1$ (since the regularisation term of $b, h^{d}$ is smaller). In Test 2 Kačur algorithm converges in even less iterations than our scheme.

We turn now to a case where $u$ is discontinuous. On the same space-time domain, take the odd function $b$ defined on $\mathbb{R}^{+}$by $b(r)=1$ for $r>1$, and $b(r)=-r^{2}+2 r$ for $0 \leq r<1$. With $f(x, t)=40 t$ for $t<0.5$, and $-40 t$ for $t \geq 0.5$, and $u_{0}=v_{0}=0$. The point is that the discontinuity of $f$ occurs when $(E P$ ) is elliptic (because $u>1)$, so $u$ has a jump too, at $t=0.5$, and falls into $[-1,1]$ where the equation is parabolic. There is a parabolic transition while $u$ remains in $[-1,1]$, and then the equation becomes elliptic so the rapidly varying $f$ acts directly on $u$. Trying to make algorithm of [11] converging is hard, we had to rely on relaxation with $\alpha=0.9$ and with a great regularisation term $h^{d}$ with $d=0.2$, or it does not converge for $t=0.5$. Then there is a large smoothing of the profile induced by the method. Thus our method can handle discontinuities in $u$ that seem hard to compute with the other algorithm. In Figure 4 the superposed graphs of $u$ (resp. $b(u))$ obtain by the two methods are plotted.

\section{Conclusion AND POSSIBle EXTENSIONS}

Note that as indicated before, the time step needs not to be constant in the definition of mild solution, leading to an adaptative time step method. This may be interesting, when the solution is expected to present a jump (in $u$ ) at a certain time. Indeed in this case one could operate thiner time discretization around this discontinuity time. 

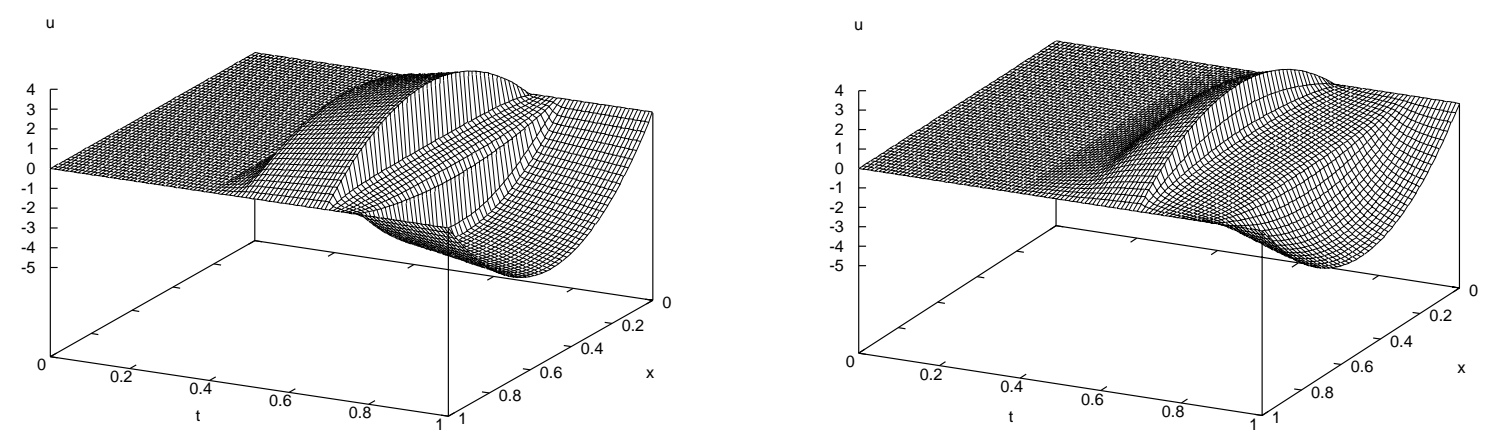

Figure 4. When $u$ is discontinuous, our algorithm (left) and the method of Jäger and Kačur (right).

One could also study the more explicit scheme

$$
\left(S_{\exp }\right) \quad\left\{\begin{array}{l}
\text { Let } u_{n+1}^{0}=u_{n}, \text { solve for } k=0,1, \ldots, \\
u_{n+1}^{k+1}-\rho \operatorname{div} a\left(u_{n}, \nabla u_{n+1}^{k+1}\right)=\lambda_{k} u_{n+1}^{k}-\frac{\rho}{h}\left(b\left(\lambda_{k} u_{n+1}^{k}\right)-b\left(u_{n}\right)\right)+\rho f_{n+1} .
\end{array}\right.
$$

With more regularity on $a$, one could study its convergence toward a weak solution of $(E P)$.

Another possible extension is to elliptic-parabolic equations of the following form:

$$
M\left(\frac{\mathrm{d} u}{\mathrm{~d} t}\right)-\operatorname{div} a(u, \nabla u)=f
$$

to which our algorithm seems to adapt without difficulty. In this case the iteration would be

$$
u_{n+1}^{k+1}-\rho \operatorname{div} a\left(u_{n+1}^{k+1}, \nabla u_{n+1}^{k+1}\right)=\lambda_{k} u_{n+1}^{k}-\rho M\left(\frac{\lambda_{k} u_{n+1}^{k}-u_{n}}{h}\right)+\rho f_{n+1} .
$$

Note that existence results for this class of equation have been obtained in $[3,7,8]$ for more restrictive vector field $a$. A work is in progress in this direction.

\section{REFERENCES}

[1] H.W. Alt and S. Luckhaus, Quasilinear Elliptic-Parabolic Differential Equations. Math. Z. 183 (1983) 311-341.

[2] H. Bauschke, The approximation of fixed points of composition of nonexpansive mappings in Hilbert space. J. Math. Anal. Appl. 202 (1996) 150-159.

[3] Ph. Bénilan and K. Ha, Equation d'évolution du type $(\mathrm{d} u / \mathrm{d} t)+\beta \partial \varphi(u) \ni 0$ dans $L^{\infty}(\Omega)$. C.R. Acad. Sci. Paris Sér. A 281 (1975) 947-950.

[4] A. Berger, H. Brézis and J. Rogers, A numerical method for solving the problem $u_{t}-\Delta f(u)=0$. RAIRO Anal. Numér. 13 (1979) 297-312.

[5] Ph. Bénilan and P. Wittbold, On mild and weak solutions of elliptic-parabolic problems. Adv. Differential Equations 1 (1996) 1053-1073.

[6] Ph. Bénilan and P. Wittbold, Sur un problème parabolique-elliptique. ESAIM: M2AN 33 (1999) 121-127.

[7] P. Colli, On Some Doubly Nonlinear Evolution Equations in Banach Spaces. Technical Report 775, Università di Pavia, Istituto di Analisi Numerica (1991).

[8] P. Colli and A. Visintin, On a class of doubly nonlinear evolution equations. Comm. Partial Differential Equations 15 (1990) 737-756.

[9] B. Halpern, Fixed points of nonexpansive mappings. Bull. Amer. Math. Soc. 73 (1967) 957-961. 
[10] W. Jäger and J. Kačur, Solution of Porous Medium Type Systems by Linear Approximation Schemes. Numer. Math. 60 (1991) 407-427.

[11] W. Jäger and J. Kačur, Solution of Doubly Nonlinear and Degenerate Parabolic Problems by Relaxation Schemes. RAIRO Modél. Math. Anal. Numér. 29 (1995) 605-627.

[12] J. Kačur, Solution of Some Free Boundary Problems by Relaxation Schemes. SIAM J. Numer. Anal. 36 (1999) $290-316$.

[13] J. Kačur, A. Handlovičová and M. Kačurová, Solution of Nonlinear Diffusion Problems by Linear Approximation Schemes. SIAM J. Numer. Anal. 30 (1993) 1703-1722.

[14] J.-L. Lions, Quelques Méthodes de Résolution des Problèmes aux Limites Non Linéaires. Dunod (1969).

[15] P.-L. Lions, Approximation de points fixes de contractions. C.R. Acad. Sci. Paris Sér. A. 284 (1977) 1357-1359.

[16] E. Magenes, R.H. Nochetto and C. Verdi, Energy Error Estimates for a Linear Scheme to Approximate Nonlinear Parabolic Problems. RAIRO Modél. Math. Anal. Numér. 21 (1987) 655-678.

[17] E. Maitre, Sur une classe d'équations à double non linéarité : application à la simulation numérique d'un écoulement visqueux compressible. Thèse, Université Grenoble I (1997).

[18] E. Maitre and P. Witomski, A pseudomonotonicity adapted to doubly nonlinear elliptic-parabolic equations. Nonlinear Anal. TMA (to appear).

[19] F. Otto, $L^{1}$-Contraction and Uniqueness for Quasilinear Elliptic-Parabolic Equations. J. Differential Equations 131 (1996) $20-38$.

[20] F. Simondon, Sur l'équation $b(u)_{t}-a(u, \nabla u)=0$ par la méthode des semi-groupes dans $L^{1}$. Séminaire d'analyse non linéaire, Laboratoire de Mathématiques de Besançon (1984).

To access this journal online: www.edpsciences.org 